\title{
Clinical Outcomes of Monofocal Toric IOLs Using Digital Tracking and Intraoperative Aberrometry
}

John F Blaylock'

Brad J Hall (D) ${ }^{2}$

'Valley Laser Eye Centre, Abbotsford, BC, V2S 3RI, Canada; ${ }^{2}$ Sengi, Penniac, NB, E3A 8X8, Canada
Correspondence: Brad J Hall Sengi, 473 Route 628, Penniac, NB, E3A8X8, Canada

Tel +I 8882558680

Email bhall@sengiclinical.com
Purpose: To evaluate clinical outcomes of a toric IOL using digital tracking (DT) and intraoperative aberrometry (IA).

Methods: This was a retrospective, single surgeon study examining 151 eyes of 106 patients. Inclusion criteria were subjects who presented with visually significant cataracts (or as a candidate for clear lens extraction) and were implanted with a toric intraocular lens. Spherical equivalent prediction errors for IA and preoperative planning were calculated and compared. Preoperative and postoperative refractive data and monocular uncorrected distance visual acuity (UDVA) and corrected distance visual acuity (CDVA) were also collected at 3 months postoperatively.

Results: Postoperative actual residual refractive astigmatism with IA was $0.50 \mathrm{D}$ or less in 140 eyes $(92.8 \%)$ and was $0.50 \mathrm{D}$ or less in 88 eyes $(58.3 \%)$ with back-calculations based on preoperative planning. The absolute spherical equivalent prediction error was $0.50 \mathrm{D}$ or less in 135 eyes $(89.4 \%)$ for IA compared to 123 eyes $(85.4 \%)$ for preoperative planning. Postoperative monocular UDVA was 0.10 logMAR or better in 124 eyes $(82.1 \%)$ and 0.00 $\log$ MAR or better in 90 eyes $(59.6 \%)$. Postoperative CDVA was $0.10 \log$ MAR or better in 147 eyes (97.4\%) and 134 eyes $(88.7 \%)$ were $0.00 \operatorname{logMAR}$ or better.

Conclusion: The results demonstrate that toric implantation with DT and IA can provide excellent refractive and visual outcomes.

Keywords: toric IOL, cataract surgery, intraoperative aberrometry

\section{Plain Language Summary}

During cataract surgery, the natural opaque lens is replaced with an artificial intraocular lens. If the front of the eye (cornea) is curved differently in one direction compared to the other (eg, the cornea is shaped like an American football instead of a basketball), that eye has astigmatism which may also be corrected at the time of cataract surgery. A type of intraocular lens (IOL), known as toric lenses, can be used to correct astigmatism. Selecting the appropriate power for a toric IOL requires accurate measurements of the eye, including eye length and the curvature of the cornea.

During cataract surgery, a surgeon can use an intraoperative aberrometer (IA) to perform measurements of the eye and refine IOL power selection. This study was a chart review, designed to compare refractive outcomes using an IA to theoretical refractive outcomes had the IA not been used. Astigmatism was reduced where an IA was used compared to theoretical results had the IA not been used. These results indicate that there are significant benefits to using IA during cataract surgery to refine or confirm IOL spherical as well as cylindrical power selection.

\section{Introduction}

Cataract surgery is also a refractive procedure, and increasing numbers of patients are expecting to reduce spectacle dependence after intraocular lens (IOL) 
implantation. Postoperative residual astigmatism (PRA) is a major factor that can lead to suboptimal visual outcomes. Distance visual acuity could be reduced by as much as 1.5 lines for every diopter of PRA. ${ }^{1}$ Toric IOLs can be used to correct astigmatism and have been reported to provide superior visual outcomes compared to relaxing incisions. ${ }^{2}$ The effectiveness of toric IOLs will depend on accurate power calculations, and accurate determination of the axis of placement. Modern toric intraocular lens formulas allow fairly accurate predictions of postoperative refraction, but there remains room for improvement. The absolute prediction error (for PRA) of toric intraocular lens formulas has been reported to be greater than $0.5 \mathrm{D}$ in $35-46 \%$ of eyes depending on the formula that is used. ${ }^{3}$

Intraoperative aberrometry (IA) can be thought of as a surgical refractometer. It is used to measure aphakic eyes during cataract surgery, which allows for validating or refining of the IOL planned power from preoperative calculations. Additionally, IA is used to measure pseudophakic eyes during cataract surgery to determine the optimal toric placement. ${ }^{4}$ The ORA System (Alcon Vision, LLC, Fort Worth, TX, USA) is currently the only IA device commercially available. Studies of the ORA system have so far reported mixed results for predicting postoperative spherical equivalent (SE). A large investigation of more than 30,000 eyes compared IA to preoperative planning and reported that IA had a lower absolute prediction error (SE). ${ }^{5}$ A separate study using low-power toric IOLs also reported that IA resulted in a lower prediction error (SE) than preoperative planning. ${ }^{6}$ However, other studies have reported no significant differences in prediction error (SE) between IA and preoperative planning. ${ }^{7-9}$

Previous studies investigating PRA using IA and preoperative planning have reported significantly reduced PRA with IA compared to preoperative planning. ${ }^{4,10} \mathrm{We}$ recently published a comparison of the postoperative residual astigmatism (PRA) of the PanOptix trifocal toric (Alcon Vision, LLC, Fort Worth, TX, USA), which followed the ORA recommendation, to back-calculated residual astigmatism (BRA; determined via vector analysis using preoperative cylinder power) that would have resulted using the cylinder power calculated preoperatively. ${ }^{11}$ We reported that the proportion of eyes with PRA $\leq 0.50 \mathrm{D}$ was significantly higher using IA (97.8\%) versus the preoperative planned cylinder power $(87.3 \%)$, and that the absolute prediction error (SE) was significantly lower with IA compared to preoperative planning. ${ }^{11}$ The purpose of this investigation is to evaluate clinical outcomes of a monofocal toric IOL using digital tracking (DT) and IA and compare it to back-calculated refractive outcomes, which simulate implantation of the preoperative planned power.

\section{Patients and Methods}

This was a retrospective chart review of clinical outcomes at a single site of an acrylic monofocal toric IOL with DT and IA utilized during implantation. Patient verbal informed consent was obtained for the use of chart data, and all extracted chart data were de-identified. As this was a retrospective study, the institutional review board approved a waiver of documentation of consent. The study was approved by an institutional review board (Advarra IRB, Aurora, ON, Canada, \#Pro00045286) and conducted in compliance with International Harmonization (ICH) guidelines, Good Clinical Practice (GCP), and the tenets of the Declaration of Helsinki.

Eligible subjects were those who presented with visually significant cataracts or as a candidate for clear lens extraction, and were implanted with a monofocal toric intraocular lens. Subjects were excluded if they had ocular comorbidity that may affect post-operative visual acuity, irregular corneal astigmatism, keratoconus, or previously underwent refractive surgery.

Data records of monofocal lens implantations between 2016 and 2019 were used to identify subjects that fit the inclusion and exclusion criteria above. A total of 151 eyes (of 106 patients) were identified that fit the inclusion and exclusion criteria. De-identified data included preoperative and postoperative data on sex, refractive error, and visual acuity (UDVA, CDVA). Visual acuities were recorded in Snellen and converted to the equivalent log of the minimum angle of resolution (logMAR) notation for statistical analysis.

Preoperative biometry measurements were performed using the IOL Master 500 (Carl Zeiss Meditec, Jena, Germany). The IOL Master 500 measures only the corneal front surface. Preoperative topography measurements were performed using the Atlas 9000 (Carl Zeiss Meditec, Jena, Germany). Tomography measurement was performed using the Pentacam (Oculus, Wetzlar, Germany). Preoperative measurements were input into the AlconBarrett Toric online calculator to determine IOL power. ${ }^{12}$ Patients who were eligible for femtosecond laser assisted cataract surgery (FLACS) were given the choice of FLACS or conventional phacoemulsification. With 
FLACS, a LenSx femtosecond laser (Alcon Vision, LLC, Fort Worth, TX, USA) was used for corneal incisions, capsulotomy, and lens fragmentation. Patients who were not eligible for or did not opt for FLACS were converted to conventional phacoemulsification. Image-guided digital tracking was performed by the VERION System (Alcon Vision, LLC, Fort Worth, TX, USA). Intraoperative aberrometry was performed using the ORA System with Verifeye + (Alcon Vision, LLC, Fort Worth, TX, USA) to determine IOL power, cylinder power, and final axis of placement. The ORA system measures both the corneal front and back surfaces. The surgeon's usual standard of care for patients implanted with IOLs was the postoperative regimen. Acrysof IQ toric IOL models were implanted (SN6AT2, SN6AT3, SN6AT4, SN6AT5, SN6AT6, SN6AT7, SN6AT8, SN6AT9; Alcon Vision, LLC, Fort Worth, TX, USA).

The primary outcome measure of interest was the postoperative manifest refraction and specifically the percentage of eyes in which the post-op MRSE resulted in an absolute prediction error of $0.50 \mathrm{D}$ or less at the 3 month postoperative visit. Secondary endpoints included backcalculated postoperative residual refractive astigmatism, monocular uncorrected visual acuity at distance (UDVA; $6 \mathrm{~m}$ ) and corrected distance visual acuity (CDVA).

The power suggested by preoperative planning and IA was not always the same power. When there was a difference, the power suggested by IA was usually preferred. Back-calculations were also performed to simulate the refractive error had the preoperative power been selected and not the power suggested by IA. Hill et al ${ }^{13}$ first described back-calculations to simulate the removal of the implanted IOL and replacement with a different IOL power. Back-calculated postoperative refractive astigmatism is a combination of 4 vectors: preoperative corneal astigmatism, surgically induced astigmatism, random surgical effects, and the IOL cylinder power. Backcalculations are used to mathematically replace the IOL vector with another. In this study, we mathematically replaced the IA cylinder power with the preoperative planned cylinder power to investigate the differences in refractive error between the two suggested powers. Backcalculations were performed by vector addition, as described in depth by Alpins. ${ }^{14}$ The first vector was the absolute difference in cylinder power at the corneal plane between the implanted and suggested IOLs, and the preoperative steep axis. The second vector was the postoperative cylinder power and axis. Back-calculations were also
Table I Preoperative Patient Demographics

\begin{tabular}{|l|c|}
\hline Parameter & Mean \pm SD (Range) \\
\hline Number of Eyes (patients) & I5I (106) \\
\hline Post-Operative Visit (days) & $71.9 \pm 27.9(29-176)$ \\
\hline Age (yr) & $70.6 \pm 10.0(22-91)$ \\
\hline Sex & 48 \\
Male (n) & 58 \\
Female (n) & $24.12 \pm 1.57(21.09-29.81)$ \\
\hline Axial Length (mm) & $1.12 \pm 0.75(0.05-3.99)$ \\
\hline Cylinder (D) &
\end{tabular}

performed to simulate spherical equivalent for suggested IA and preoperative planned powers in cases where they differed from the implanted power.

Statistical analyses were performed using the software program R (version 4.0.3; The R Foundation for Statistical Computing, Vienna, Austria). Differences in continuous variables were compared using a paired $t$-test for two continuous variables or an analysis of variance (ANOVA) for 3 or more groups. A Wilcoxon signed-rank test was used where data were not normally distributed. A chi-square statistic was used for analysis of categorical variables. In all cases, $\mathrm{p} \leq 0.05$ was considered significant.

\section{Results}

The chart review identified 151 eyes of 106 patients for inclusion in this study. Table 1 summarizes the demographic and preoperative data. The average age of patients in this study was $70.6 \pm 10.0$. Our study included similar numbers of males (48) and females (58). Of the 151 eyes in this study, 103 eyes $(68.2 \%)$ received FLACS, while 48 eyes $(31.8 \%)$ had conventional phacoemulsification.

The distribution of the postoperative residual astigmatism (PRA) for IA compared to back-calculated residual astigmatism (BRA) is shown in Figure 1. For IA, 131 eyes (86.8\%) and 140 eyes (92.8\%) had PRA of $0.25 \mathrm{D}$ or less and $0.50 \mathrm{D}$ or less, respectively. This was higher compared to BRA, where 55 eyes (36.4\%) and 88 eyes $(58.3 \%)$ had BRA of $0.25 \mathrm{D}$ or less and $0.50 \mathrm{D}$ or less, respectively. Additionally, BRA was greater than $1.0 \mathrm{D}$ in 17 eyes $(11.3 \%)$, compared to 4 eyes $(2.6 \%)$ for PRA.

Double-angle vector plots for preoperative astigmatism, postoperative astigmatism for IA (PRA), and postoperative astigmatism for back-calculations based on preoperative planning (BRA) are shown in Figure 2. The 


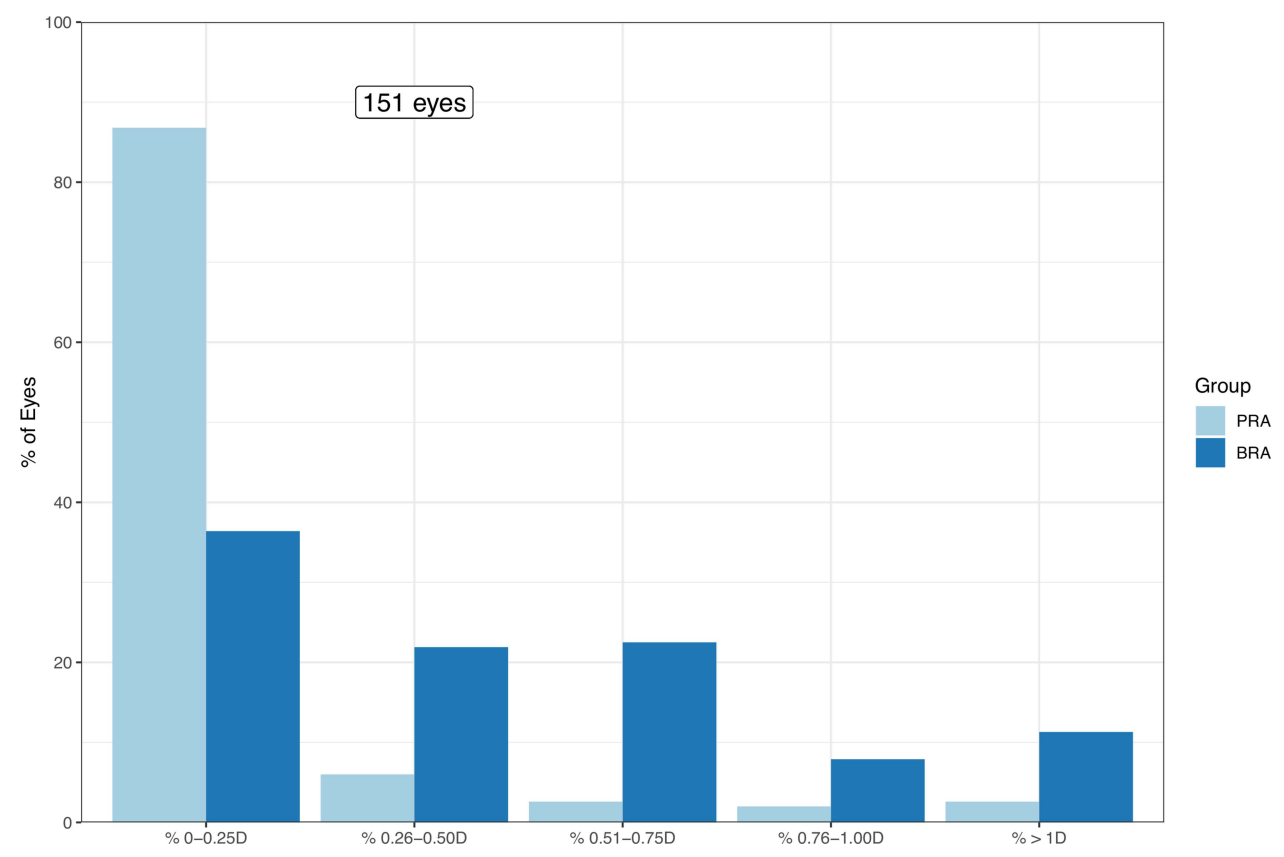

Figure I Postoperative residual astigmatism in eyes using intraoperative aberrometry (PRA) and preoperative planned power (BRA).

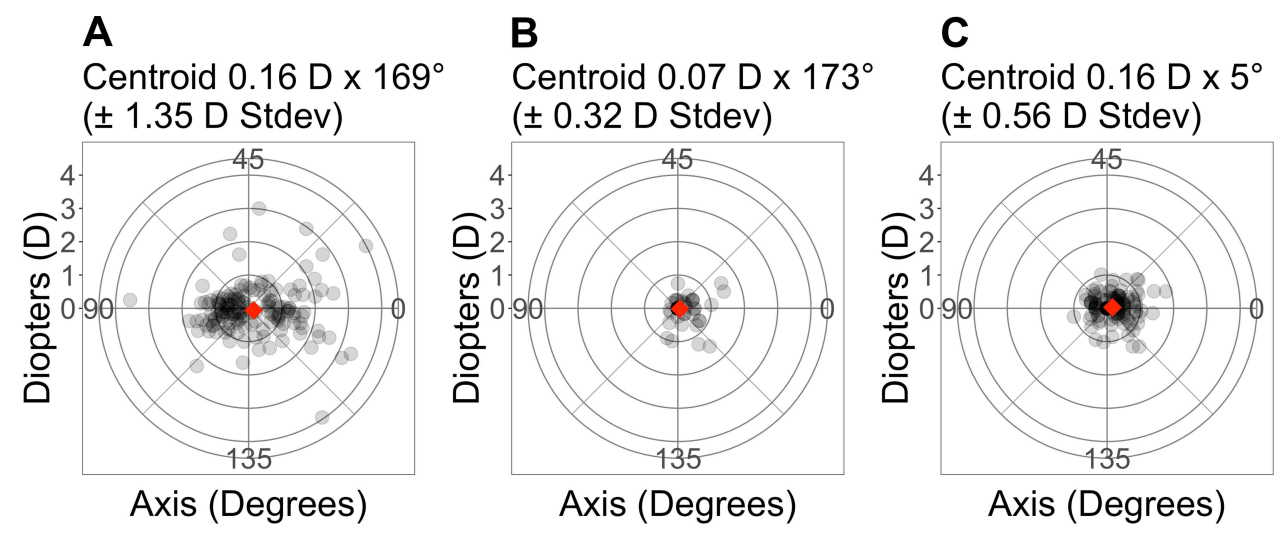

Figure 2 Double-angle vector plots of astigmatism vectors for $(\mathbf{A})$ preoperative, $(\mathbf{B})$ intraoperative aberrometry, and $(\mathbf{C})$ preoperative planned power. Each ring represents I D. The diamond represents the centroid. $\mathrm{N}=|5|$ eyes.

standard deviation for PRA and BRA, 0.32 D and 0.56 D, respectively, were lower compared to the preoperative astigmatism $(1.35 \mathrm{D})$. The standard deviation for PRA was also lower than that of the BRA.

A comparison of the postoperative refractive absolute prediction errors (SE) for IA suggested power and preoperative planned power is shown in Figure 3. For IA, the number of eyes with absolute prediction errors of 0.25 $\mathrm{D}$ or less and $0.50 \mathrm{D}$ or less were $111(73.5 \%)$ and 135 (89.4\%), respectively. By comparison, with preoperative planned power, the number of eyes with absolute prediction errors (SE) of $0.25 \mathrm{D}$ or less and $0.50 \mathrm{D}$ or less were
$76(50.3 \%)$ and 129 (85.4\%), respectively. IA demonstrated a higher proportion of eyes with absolute prediction errors (SE) of $0.25 \mathrm{D}$ or less (difference of 35 eyes [23.2\%]) and $0.50 \mathrm{D}$ or less (difference of 6 eyes [4.0\%], $\mathrm{p}<0.001)$ compared to preoperative planned power. In 85 cases $(56.3 \%)$, IA and preoperative planned power suggested different spherical powers. Of these, the power suggested by IA was selected in 62 eyes $(72.9 \%)$, preoperative planned power was selected in 13 eyes $(15.3 \%)$, and neither the IA nor the preoperative planned power was selected in 10 eyes (11.8\%). Overall, in cases where the preoperative planned power was different than the IA 


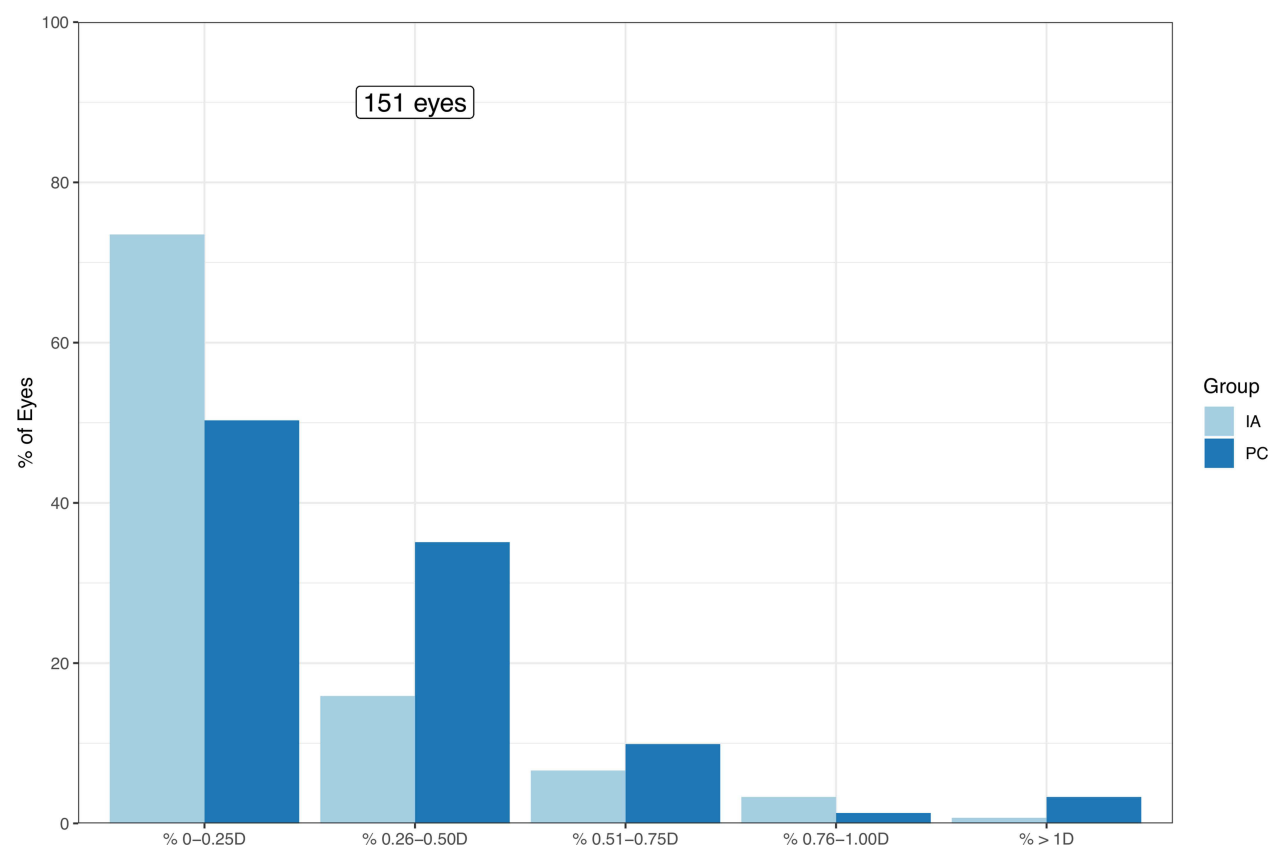

Figure 3 Postoperative refractive prediction errors in eyes using intraoperative aberrometry (IA) and preoperative planned power (PC).

suggested power (85/151 eyes), the proportion of eyes with absolute prediction errors (SE) of $0.25 \mathrm{D}$ or less and $0.50 \mathrm{D}$ or less was significantly higher $(\mathrm{p}<0.001)$ with IA (60 eyes [70.6\%] and 74 eyes [87.1\%]) than with the preoperative planned power (32 eyes [37.6\%] and 67 eyes [78.8\%]).

Figure 4 shows the standard graphs for reporting refractive outcomes. Monocular UDVA and CDVA were $20 / 25(0.10 \log$ MAR $)$ or better in 124 eyes $(82.1 \%)$ and 147 eyes (97.4\%), respectively. All eyes had CDVA of 20/ 40 (0.30 logMAR) or better. We did not find any statistically significant differences in visual acuities between lens models (SN60T2-T9, p > 0.05).

A comparison of the refractive outcomes in implanted eyes with the power suggested by IA to simulations based on the preoperative planned power is shown in Table 2. The preoperative planned power and the IA suggested powers were different spherical and toric powers in 85 eyes (56.3\%) and 90 eyes (59.6\%), respectively. Of these, IA recommended a toric IOL where the preoperative planning did not in 16 eyes $(10.6 \%)$. There were no cases where preoperative planning suggested a toric IOL and IA did not. Mean absolute prediction error was significantly higher in backcalculations based on the preoperative planned power $(0.28 \pm 0.27 \mathrm{D})$ compared to IA $(0.21 \pm 0.21 \mathrm{D}, \mathrm{P}<$ $0.001)$. Mean PRA was also significantly lower $(0.13 \pm$
$0.31 \mathrm{D})$ compared to mean BRA $(0.43 \pm 0.40 \mathrm{D}, \mathrm{P}<$ 0.001). Following the recommendation by IA compared to preoperative calculations resulted in equal residual astigmatism in 61 eyes (40.4\%), lower residual astigmatism in 81 eyes (53.6\%), and higher residual astigmatism in 9 eyes $(6 \%)$.

\section{Discussion}

To achieve high patient satisfaction after toric IOL implantation, both the postoperative astigmatism and postoperative spherical equivalent should be minimized by selecting the appropriate lens power. This study has compared lenses implanted based on IA to simulated implantations of lenses based on preoperative planning. Overall, the results of this study suggest that postoperative refractive outcomes are improved with IA compared to using the preoperative planned power.

Our study also supports the conclusion that IA can better predict postoperative astigmatism compared to preoperative calculations. The percentage of patients with postoperative astigmatism $0.5 \mathrm{D}$ or less was greater with IA (92.8\%) than with back-calculations based on preoperative calculations $(58.3 \%)$, a difference of $34.5 \%$. This difference is higher than that reported by Woodcock et $\mathrm{al}^{9}(12.6 \%)$; however, their study used multiple surgeons, differing preoperative formulas, and larger datasets, which may explain these differences. 


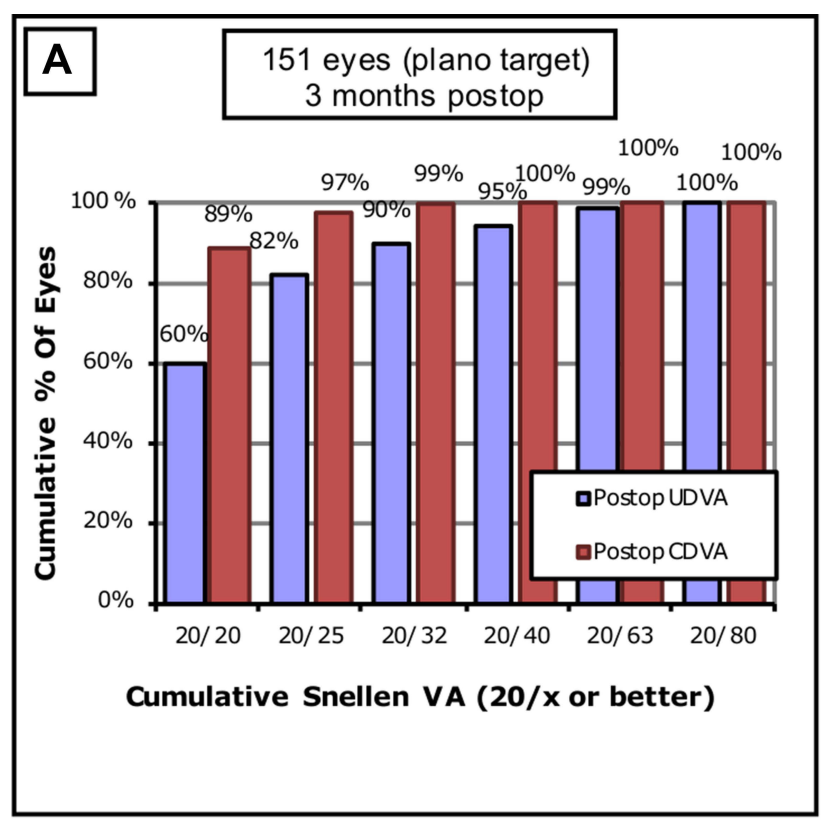

Uncorrected Distance Visual Acuity

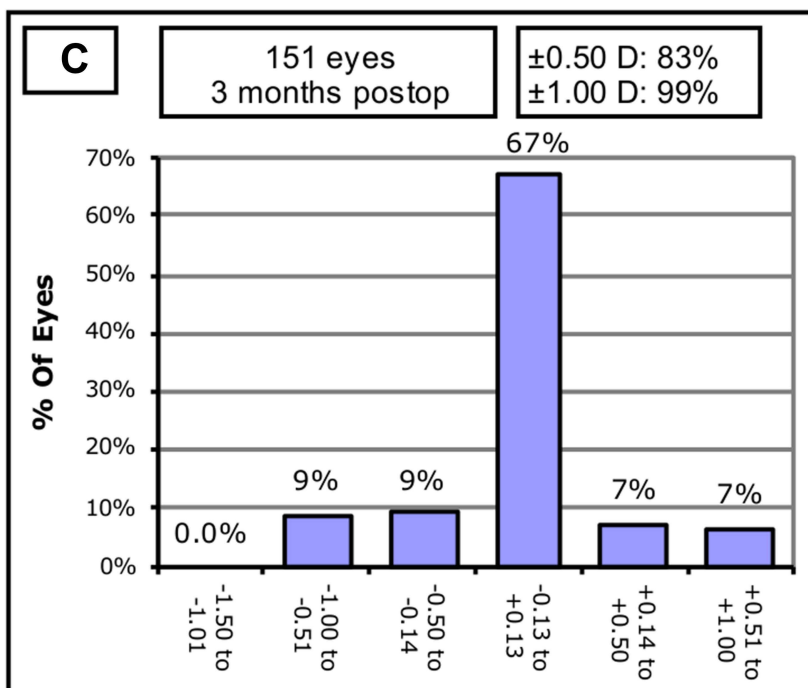

Accuracy of SEQ to Intended Target (D)

\section{Spherical Equivalent Refraction Accuracy}

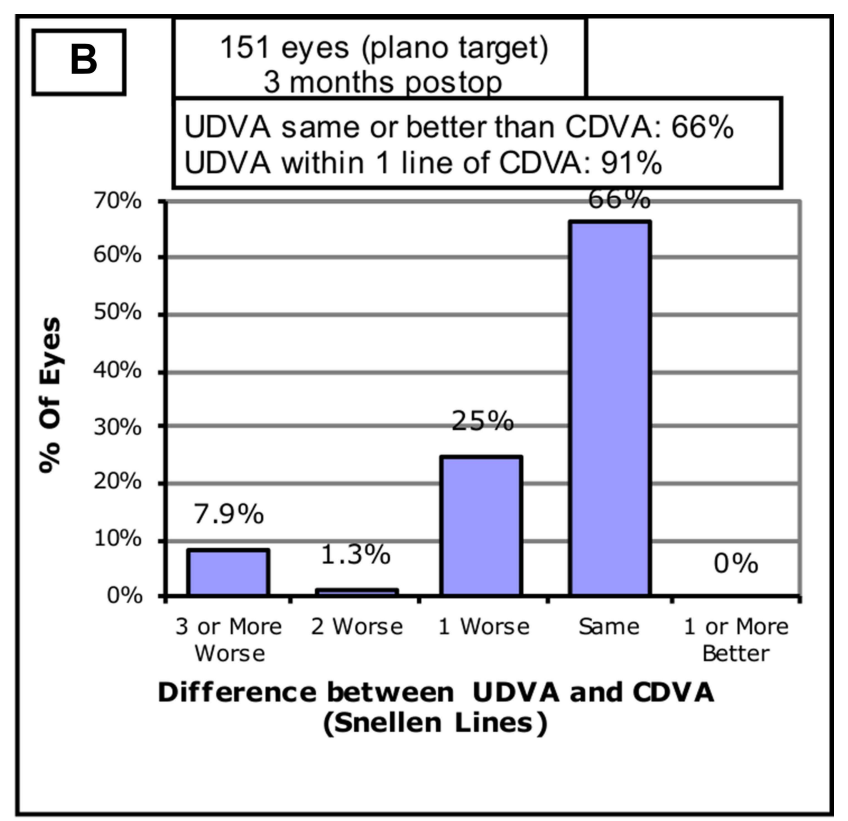

Uncorrected Distance Visual Acuity vs. Corrected Distance Visual Acuity

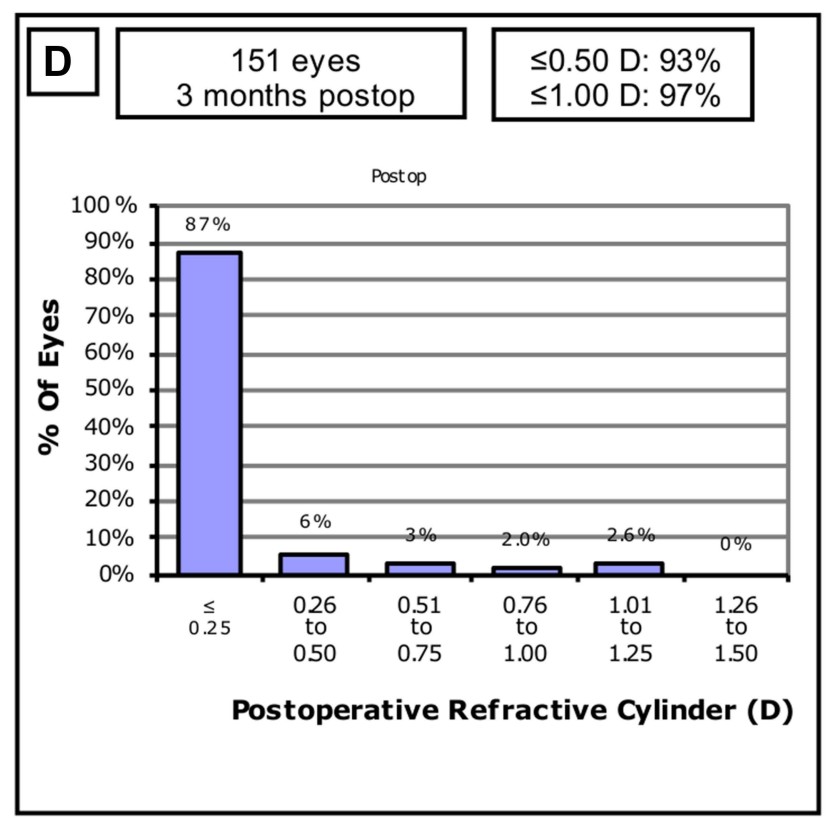

Refractive Cylinder

Figure 4 Standard graphs for reporting refractive outcomes: (A) uncorrected distance visual acuity (UDVA); (B) UDVA vs corrected distance visual acuity (CDVA); (C) spherical equivalent refraction accuracy; and (D) postoperative refractive cylinder. $\mathrm{D}=$ diopters.

Our study supports the conclusion that IA can better predict postoperative spherical equivalent compared to preoperative calculations (we used the Barrett Toric formula in this study). The percentage of eyes with a mean absolute prediction error of $0.5 \mathrm{D}$ or less was higher with IA $(89.4 \%)$ compared to preoperative calculations $(85.4 \%)$, a significant difference of $4 \%$. This difference is similar to the differences reported by Cionni et al, ${ }^{5}$ Cionni et al, ${ }^{6}$ and Woodcock et al ${ }^{9}$ of $6 \%, 6.9 \%$, and $1.8 \%$, respectively. These three studies used multiple surgeons, differing preoperative formulas, and larger datasets, which could explain the differences observed in our study. Davison and Potvin ${ }^{8}$ had a similar 
Table 2 Postoperative Refractive Outcomes

\begin{tabular}{|l|c|c|c|}
\hline Parameter & IA Mean \pm SD (Range) & $\begin{array}{c}\text { PreOP Planned Power Mean } \pm \text { SD } \\
\text { (Range) }\end{array}$ & P value \\
\hline Residual Astigmatism (D) & $0.13 \pm 0.31(0-1.50)$ & $0.43 \pm 0.40(0-1.84)$ & $<0.00 \mathrm{I}$ \\
Residual SE (D) & $-0.07 \pm 0.37(-2.16-0.75)$ & $0.08 \pm 0.42(-2.2-1.1)$ & $<0.00 \mathrm{I}$ \\
Absolute Residual SE (D) & $0.19 \pm 0.32(0-2.16)$ & $0.29 \pm 0.31(0-2.16)$ & $<0.00 \mathrm{I}$ \\
Absolute Prediction Error (SE) & $0.21 \pm 0.21(0-1.0 \mathrm{I})$ & $0.28 \pm 0.28(0-1.5 \mathrm{I})$ & $<0.00 \mathrm{I}$ \\
\hline
\end{tabular}

Abbreviations: IA, Intraoperative Aberrometry; PreOP, Preoperative; SE, spherical equivalent.

sample size to our study and reported a difference of $8.1 \%$ between IA and preoperative calculations, but this was not statistically significant. Our results are higher than that reported by Raufi et al, ${ }^{7}$ who observed a difference of $1-$ $2 \%$ in favour of preoperative calculations, but this was not statistically significant.

The implementation of IA is not meant to replace a surgeon's judgement, but to provide more information and enhance decision making. In our study, IA and preoperative planning recommended the same spherical and cylinder powers in $43.7 \%(66 / 151)$ and $40.4 \%(61 / 151)$ of cases, respectively. Where the recommendations differed, however, following the IA recommendation resulted in better refractive outcomes than following the preoperative planning recommendation.

Achieving the refractive target with the primary surgery benefits both patients and refractive surgeons. Patients are more likely to be satisfied with their visual outcomes and would not have to undergo additional enhancement procedures. Based on the results of this study, by using IA surgeons may on average reduce the number of enhancement procedures following toric IOL implantation from 42 eyes per 100 to 7 eyes per 100 .

A limitation of this study is that the IOL Maser 500 measures only the corneal front surface, whereas the ORA system measures both the front and back surfaces. This does provide a methodological advantage to IA compared to preoperative calculations, though it is unlikely that this advantage would have affected the overall trends in our data. A study by Fabian and Wehner ${ }^{15}$ found a difference of approximately $0.02 \mathrm{D}$ in absolute prediction error of sphere and cylinder with the Barrett Formulas when comparing the IOL Master 700 with and without Total Keratometry. Nevertheless, a future study directly comparing IA to biometers that use total keratometry is warranted.

Another limitation of this study is that predictions of postoperative astigmatism with IA and preoperative planning were not collected, and thus we cannot present any data regarding the prediction errors for postoperative astigmatism. Kane et $\mathrm{al}^{3}$ recently published a large study comparing the prediction errors for postoperative astigmatism for many toric IOL formulas and found that the best performing formula still had cylinder prediction errors of greater than $0.5 \mathrm{D}$ in $34.4 \%$ of eyes. A future study comparing the prediction errors for postoperative astigmatism with IA to preoperative planning formulas is needed. An additional limitation is that this is a retrospective study of 1 surgeon at a single site. This does provide internal validity, but may reduce applicability to other surgeons. We also acknowledge that using IA is part of the surgeon's standard of treatment, which may introduce some bias.

\section{Conclusions}

The results of this study demonstrate that implantation with DT and IA can provide excellent refractive and visual outcomes. These results also support the conclusion that IA can better predict postoperative spherical equivalent and astigmatic outcomes, as the mean postoperative astigmatism and percentage of eyes within $0.50 \mathrm{D}$ of postoperative astigmatism was higher with IA compared to preoperative calculations.

\section{Funding}

This study was supported with an investigator-initiated study grant (60521397) from Alcon Vision, LLC, Fort Worth, TX, USA.

\section{Disclosure}

JF Blaylock is a consultant to Alcon Vision, LLC. BJ Hall reports personal fees from Valley Laser Eye Centre. The authors report no other conflicts of interest in this work.

\section{References}

1. Lehmann RP, Houtman DM. Visual performance in cataract patients with low levels of postoperative astigmatism: full correction versus spherical equivalent correction. Clin Ophthalmol. 2012;6:333-338.

2. Kessel L, Andresen J, Tendal B, Erngaard D, Flesner P, Hjortdal J. Toric intraocular lenses in the correction of astigmatism during cataract surgery: a systematic review and meta-analysis. Ophthalmology. 2016;123:275-286. 
3. Kane JX, Connell B. A comparison of the accuracy of 6 modern toric intraocular lens formulas. Ophthalmology. 2020;127:1472-1486.

4. Hatch KM, Woodcock EC, Talamo JH. Intraocular lens power selection and positioning with and without intraoperative aberrometry. $J$ Refract Surg. 2015;31:237-242.

5. Cionni RJ, Dimalanta R, Breen M, Hamilton C. A large retrospective database analysis comparing outcomes of intraoperative aberrometry with conventional preoperative planning. J Cataract Refract Surg. 2018;44:1230-1235.

6. Cionni RJ, Breen M, Hamilton C, Williams R. Retrospective analysis of an intraoperative aberrometry database: a study investigating absolute prediction in eyes implanted with low cylinder power toric intraocular lenses. Clin Ophthalmol. 2019;13:1485-1492.

7. Raufi N, James C, Kuo A, Vann R. Intraoperative aberrometry vs modern preoperative formulas in predicting intraocular lens power. $J$ Cataract Refract Surg. 2020;46:857-861.

8. Davison JA, Potvin R. Preoperative measurement vs intraoperative aberrometry for the selection of intraocular lens sphere power in normal eyes. Clin Ophthalmol. 2017;11:923-929.

9. Woodcock MG, Lehmann R, Cionni RJ, Breen M, Scott MC. Intraoperative aberrometry versus standard preoperative biometry and a toric IOL calculator for bilateral toric IOL implantation with a femtosecond laser: one-month results. J Cataract Refract Surg. 2016;42:817-825.
10. Blehm C, Potvin R. Pseudophakic astigmatism reduction with femtosecond laser-assisted corneal arcuate incisions: a pilot study. Clin Ophthalmol. 2017;11:201-207.

11. Blaylock JF, Hall B. Astigmatic results of a diffractive trifocal Toric IOL following intraoperative aberrometry guidance. Clin Ophthalmol. 2020;14:4373-4378.

12. Barrett G Alcon-Barrett Online Toric Calculator. Alcon; 2019. Available from: https://www.myalcon-toriccalc.com/\#/calculator. Accessed January 24, 2021.

13. Hill W, Osher R, Cooke D, et al. Simulation of toric intraocular lens results: manual keratometry versus dual-zone automated keratometry from an integrated biometer. $J$ Cataract Refract Surg. 2011;37:2181-2187.

14. Alpins NA. A new method of analyzing vectors for changes in astigmatism. J Cataract Refract Surg. 1993;19:524-533.

15. Fabian E, Wehner W. Prediction accuracy of total keratometry compared to standard keratometry using different intraocular lens power formulas. J Refract Surg. 2019;35:362-368.
Clinical Ophthalmology

\section{Publish your work in this journal}

Clinical Ophthalmology is an international, peer-reviewed journal covering all subspecialties within ophthalmology. Key topics include: Optometry; Visual science; Pharmacology and drug therapy in eye diseases; Basic Sciences; Primary and Secondary eye care; Patient Safety and Quality of Care Improvements. This journal is indexed on PubMed

\section{Dovepress}

Central and CAS, and is the official journal of The Society of Clinical Ophthalmology (SCO). The manuscript management system is completely online and includes a very quick and fair peer-review system, which is all easy to use. Visit http://www.dovepress.com/ testimonials.php to read real quotes from published authors. 